\title{
Dielectric and Microwave Properties of Siloxane Rubber/Carbon Black Nanocomposites and Their Correlation
}

\author{
Omar A. Al-Hartomy, ${ }^{1,2}$ Falleh Al-Solamy, ${ }^{3}$ Ahmed Al-Ghamdi, ${ }^{2}$ Nikolay Dishovsky, ${ }^{4}$ \\ Vladimir Iliev, ${ }^{5}$ and Farid El-Tantawy ${ }^{6}$ \\ ${ }^{1}$ Physics Department, Faculty of Science, University of Tabuk, 71491 Tabuk, Saudi Arabia \\ ${ }^{2}$ Physics Department, Faculty of Sciences, King Abdulaziz University, 21589 Jeddah, Saudi Arabia \\ ${ }^{3}$ Mathematics Department, Faculty of Science, University of Tabuk, 71491 Tabuk, Saudi Arabia \\ ${ }^{4}$ Department of Polymer Engineering, University of Chemical Technology and Metallurgy, 1756 Sofia, Bulgaria \\ ${ }^{5}$ College of Telecommunications and Posts, 1700 Sofia, Bulgaria \\ ${ }^{6}$ Department of Physics, Faculty of Science, Suez Canal University, Ismailia, Egypt
}

Correspondence should be addressed to Nikolay Dishovsky, dishov@uctm.edu

Received 10 March 2011; Revised 24 May 2011; Accepted 25 May 2011

Academic Editor: Marek Cypryk

Copyright (C) 2011 Omar A. Al-Hartomy et al. This is an open access article distributed under the Creative Commons Attribution License, which permits unrestricted use, distribution, and reproduction in any medium, provided the original work is properly cited.

In this paper, the dielectric and microwave properties of carbon black/siloxane rubber-based nanocomposites have been investigated in the frequency range from $1 \mathrm{GHz}$ till $12 \mathrm{GHz}$ according to the content of carbon black and the frequency. It has been established that the increasing frequency and filler content lead to an increase in the relative permittivity and tangent of dielectric loss angle. At higher filler content, the effects become more pronounced, especially those upon dielectric loss. It has been also established that there are two well-distinguished areas in all dependences of microwave properties on frequency and filler content increasing. The first is between 1 and $8 \mathrm{GHz}$ wherein the reflection and attenuation of microwaves do not change considerably with frequency and filler content alternation while shielding effectiveness worsens. The second area is between 8 and $12 \mathrm{GHz}$ wherein the reflection and attenuation of microwaves increase drastically with the increasing frequency and filler content. Shielding effectiveness improves, too. It has been established that in all cases the degree of correlation between dielectric and microwave properties evaluated on the basis of the coefficients of correlation calculation is perfect.

\section{Introduction}

As the central processing units (CPU) of personal computers become faster, in the near future, they are projected to reach a few tens of gigahertz $(\mathrm{GHz})$. In wireless telecommunication systems, microwaves in the range of $\mathrm{GHz}$ have extensively been used. The electromagnetic interference (EMI) of unwanted waves, which cause malfunctions in electronic equipment, has been of concern up to the frequency range of $\mathrm{GHz}$. To solve the EMI problem, two methods have been suggested, the shielding and the absorbing of the electromagnetic field. In respect to shielding, most shielding materials have high conductivity. There is a possibility of electric leakage in the shielding method because the electronics have become more integrated in a limited area, and the gap between circuit units has become narrower. In addition, the conductive material setting on a circuit for the shielding of the electromagnetic field can act as an antenna that causes malfunctions in the electronic equipment. Therefore, electromagnetic absorbing materials, which are usually nonconductive materials, are known as a safer method for solving the EMI problem [1].

Siloxane elastomers are unusual synthetic polymers whose backbone is composed of an "inorganic" repeating unit, that is, silicon to oxygen bonds. In addition, the silicone atoms are attached to organic groups, such as methyl. The presence of the inorganic and organic units gives siloxanes their unique properties and thermal and chemical stability. At extreme temperatures the tensile strength, elongation, tear strength, and compression set can be far superior to 
those of conventional rubbers. Organic rubber (NR, SBR, $\mathrm{BR}$, etc.) has a carbon-to-carbon backbone which can make them susceptible to ozone, UV, heat, and other ageing factors that silicone rubber can withstand well. This makes it one of the elastomers of choice in many extreme environments [2]. Hence, siloxane-based micro- and nanocomposites have received increasing attention in the recent years [3-5]. A variety of fillers can be used in siloxane rubber to change its properties, but most of them are nonreinforcing and decrease the tensile properties. Carbon black is generally well-known reinforcing filler used to improve the mechanical properties and to prevent their ultraviolet degradation [6]. Blending siloxane rubber with carbon black gives the benefit of light weight, compared with blending ferrite in a rubber matrix. Therefore, we have focused our attention on a thin flat absorber made from blends of carbon black and siloxane rubber.

In this paper, we investigated the dielectric and microwave properties of carbon black/siloxane rubber nanocomposites in the frequency range from $1 \mathrm{GHz}$ till $12 \mathrm{GHz}$ according to the content of carbon black and the frequency. The correlation between dielectric and microwave properties has been also investigated. On the ground of the above said it could be expected that the nanocomposites based on siloxane rubber comprising carbon black would possess a number of interesting properties, opening new opportunities for their application in the field mentioned above.

\section{Experimental}

2.1. Materials. Siloxane rubber paste with high temperature cure and hardness of 30 Shore A was purchased from North Special Rubber Corporation of Hengshui, Hebei Province, China. Furnace carbon black Corax N220 (produced by Evonik) with typical average particle size of $20-25 \mathrm{~nm}$ and $\mathrm{N}_{2}$ surface area of $100-120 \mathrm{~m}^{2} / \mathrm{g}$ was used as conductive filler. The black was received from Alexandria Trade Rubber Company, Alexandria, Egypt.

2.2. Preparation of Rubber Nanocomposites. The mixing was accomplished in an open two-roll mill under identical conditions of time, temperature, and nip gap, with same sequence of mixing of all compounding ingredients to avoid the effect of processing on physical properties. Several batches with different concentration of carbon black 100/0, 90/10, 80/20, 70/30, 60/40 w.\% abbreviated as Si 0, Si 10, Si 20 , Si 30, Si 40, respectively, were prepared. The vulcanization process of the siloxane rubber-based compounds was carried out in an electrically heated hydraulic press using a special homemade mold at temperature $195^{\circ} \mathrm{C}$ and under pressure $150 \mathrm{kN} / \mathrm{m}^{2}$ for $60 \mathrm{~min}$.

\section{Characterization and Measurements}

\subsection{Microwave Properties}

3.1.1. Reflection and Attenuation. Measurements of reflection and attenuation were carried out using the measurement of output (adopted) power $P_{a}$ in the output of a measuring line without losses, where samples of materials might be included. A coaxial line was used because of the wide frequency measurement. Samples of the materials were shaped like discs with an external diameter $D=20.6 \mathrm{~mm}$, equal to the outer diameter of the coaxial line and thickness $\Delta \approx 2 \mathrm{~mm}$. The internal diameter depended on the relative dielectric permittivity of the material.

Part of the incident electromagnetic wave with power $P_{\text {in }}$ on the sample was reflected from it. The rest of the wave with power $P_{p}$ penetrated the material, so that the attenuation $L$ depended on the coefficient of reflection $|\Gamma|$. Its module was determined by a reflect meter. So attenuation was determined by

$$
L=10 \log \frac{P_{a}}{P_{p}}, \mathrm{~dB}, \quad(\mathrm{~dB} \text {-Decibels })
$$

where

$$
P_{p}=P_{\text {in. }}\left(1-|\Gamma|^{2}\right) .
$$

The following scheme was used for testing both parameters (Figure 1).

3.1.2. Shielding Effectiveness (S.E.) This parameter is defined as the sum of the reflection losses $R, \mathrm{~dB}$ and attenuation $L$, $\mathrm{dB}$ in the material [7].

It can be directly measured or determined by the calculation method on data from the measured reflectance and attenuation in the material. In the first case, as measured: incident power on the sample $P_{\text {in }}$ and adopted after the sample $P_{a}$ (Figure 1), S.E. is determined by

$$
\text { S.E. }=10 \log \frac{P_{\text {in }}}{P_{a}}, \mathrm{~dB} .
$$

In the second, if the reflection and absorption in the material are known, S.E. is determined, by definition, as

$$
\text { S.E. }=R, \mathrm{~dB}+L, \mathrm{~dB},
$$

where $R, \mathrm{~dB}$ is the attenuation due to the reflection of power at the interfaces.

\subsection{Dielectric Properties}

3.2.1. Complex Permittivity. The determination of complex permittivity was carried out at resonance method, based on cavity perturbation technique [8].

The resonance frequency of the empty cavity resonator $f_{r}$ was measured followed by measuring the shift in resonance frequency with the sample material $f_{\varepsilon}$. The dielectric constant $\varepsilon_{r}$ was calculated from the shift in resonance frequency, cavity and the sample cross sections $S_{r}$ and $S_{\varepsilon}$, respectively,

$$
\varepsilon_{r}=1+\frac{S_{r}}{2 S_{\varepsilon}} \cdot \frac{f_{r}-f_{\varepsilon}}{f_{r}} .
$$

The sample was in the form of disc with a diameter of $10 \mathrm{~mm}$ and thickness about $2 \mathrm{~mm}$. It was placed at the 


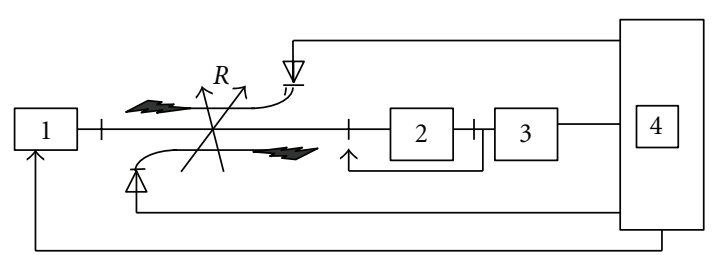

FIGURE 1: Scheme of the equipment for measuring the microwave properties 1: represent a set of generators for the whole range: HP686A and G4-79 to 82; 2: coaxial section of the deck E2M Orion, with samples of material; 3: power meter HP432A; 4: scalar reflectance meter HP416A; $R$ : Reflect meter, including (i) two directional couplers Narda 4222.16; (ii) two crystal detectors Narda 4503-N.

position of maximum electric field of the cavity. Because the thickness of the sample was not equal to the height of the resonator, a dielectric was obtained with an equivalent permittivity $\varepsilon_{e}$ where it was positioned. The equivalent permittivity $\varepsilon_{e}$ was determined by (5) where $\varepsilon_{r}$ was replaced by $\varepsilon_{e}$. Then $\varepsilon_{r}$ was determined by

$$
\varepsilon_{r} \approx \varepsilon_{e}(k+1)-k, \quad(\Delta \ll l),
$$

where $k=l / \Delta$ and $l$ is the distance from the disc to the top of the resonator, $\Delta$ is the sample thickness.

3.2.2. Loss Factor $\tan \delta$. The loss factor $\tan \delta$ was calculated from quality factor of the cavity with $Q_{\varepsilon}$ and without sample $Q_{r}$

$$
\tan \delta=\frac{1}{4 \varepsilon_{r}} \frac{S_{r}}{S_{\varepsilon}}\left(\frac{1}{Q_{\varepsilon}}-\frac{1}{Q_{r}}\right) .
$$

The measurement setup used several cavity resonators for the whole range, generators for the whole range, frequency meter, and oscilloscope.

The following scheme was used for measuring the dielectric properties (Figure 2).

3.3. Correlation Coefficient and Its Calculation [9]. Correlation coefficient is a measure of the degree of dependence between two variables. Pearson correlation coefficient is the most used and is calculated according to the formula:

$$
R=\frac{\sum_{i=1}^{n}\left(Y_{i}-\bar{Y}\right)\left(X_{i}-\bar{X}\right)}{\sqrt{\sum_{i=1}^{n}\left(Y_{i}-\bar{Y}\right)^{2} \sum_{i=1}^{n}\left(X_{i}-\bar{X}\right)^{2}}} .
$$

$X_{i}, Y_{i}$ are experimental (current) values of two variables whose correlation is searched, $\bar{X}, \bar{Y}$ are average values of the same variables.

Correlation coefficients have the following characteristics. They have no measure units, that is, do not depend on the specific units of the two variables (meters, seconds, etc.).

The degree of dependence between two variables can be between +1 and -1 , including:

$0<R<0.3$ - low degree of correlation, $0.3<R<0.5$-medium degree of correlation,

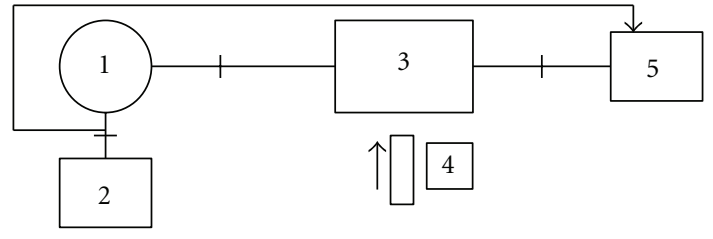

Figure 2: Scheme of the equipment for measuring the dielectric properties: 1: generators for the whole range: HP686A and G4-79 to 82 ; 2: frequency meters: $\mathrm{H}$ 532A; FS-54; 3: cavity resonator; 4 : sample; 5: oscilloscope EO 213.

$0.5<R<0.7$ - significant degree of correlation,

$0.7<R<0.9$-very high degree of correlation,

$0.9<R<1.0$ - perfect degree of correlation.

Direction of the correlation:

+ positive as one variable increases, the other variables also increase,

- negative as one variable increases, the other variables decrease.

\section{Results and Discussion}

4.1. Dielectric Properties. The dielectric properties of a material refer to the complex relative permittivity $\varepsilon^{*}=\varepsilon^{\prime}-$ $j \varepsilon^{\prime \prime}$, where $\varepsilon^{\prime}$ is the real relative dielectric permittivity $\left(\varepsilon_{r}\right)$, which is also known as the dielectric constant, whereas the imaginary part $\varepsilon^{\prime \prime}$ is the dielectric loss factor. The dielectric constant is a measure of ability of the material to be polarized or energy stored, while the loss factor is a measure of the ability of the material to heat or energy loss by absorbing energy [10].

In the present work the relative dielectric permittivity and dielectric loss have been measured at microwave frequencies in the $1-12 \mathrm{GHz}$ region (Figures 3-6).

4.1.1. Relative Dielectric Permittivity. As seen from Figure 3 the values of relative dielectric permittivity increase with the increasing frequency and content of carbon black filler in siloxane rubber. The increase follows a different mode: at same filler content the frequency dependence is almost linear; the increase is the lowest for the least filled sample while the highest increase is observed for the sample filled with $40 \mathrm{w} . \%$ of carbon black. But when the frequency is the same, the increase in the dielectric constant depends on filler content. The samples could be divided into three groups: in the first group is the nonfilled sample with the lowest dielectric constant value; to the second group belong the composites comprising 10,20, and $30 \mathrm{w} . \%$ of carbon black having very close dielectric permittivity values; the composite filled with $40 \mathrm{w} . \%$ of carbon black is in the third group which dielectric permittivity value is the highest and is superior to those of the composites from the other two groups. The difference in the values gets greater with the increasing frequency. There is almost no difference at $2 \mathrm{GHz}$; 


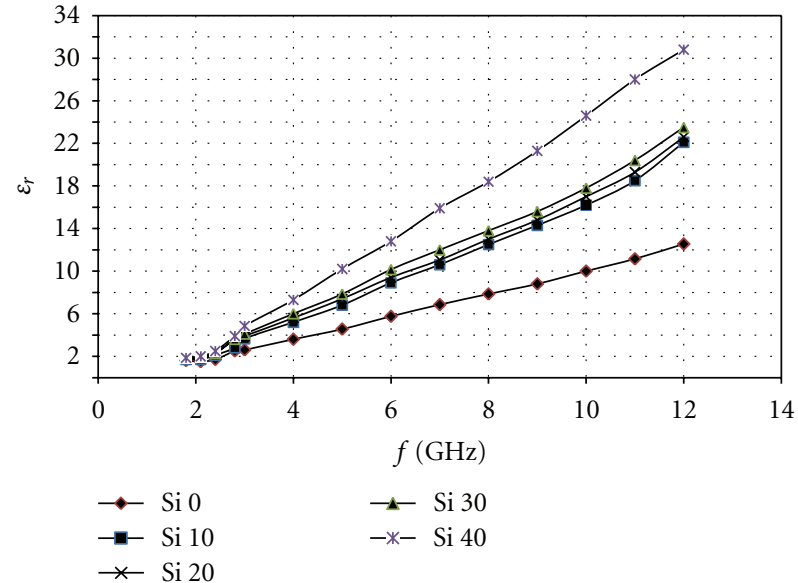

Figure 3: Frequency $(f, \mathrm{GHz})$ dependence of relative dielectric permittivity $\left(\varepsilon_{r}\right)$ at various filler content in the composites.

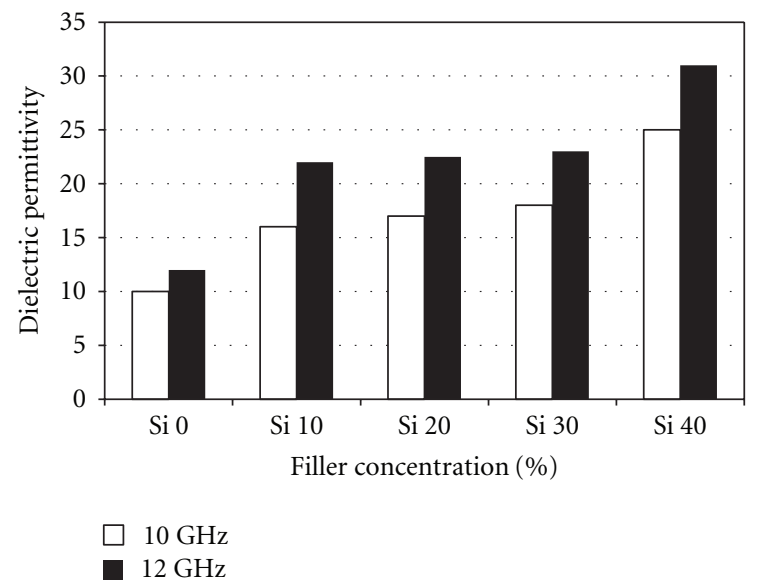

FIGURE 4: Dependence of relative dielectric permittivity on filler concentration.

at $4 \mathrm{GHz}$ it is 4 units, while at $12 \mathrm{GHz} 16$ units. Obviously, filling siloxane rubber with a significant amount of carbon black (e.g., 40 w.\%) could be used to obtain siloxane rubber composites possessing considerable dielectric permittivity values. The grouping of the composites (observed in the case of the dielectric loss as well) might be related to reaching and passing the percolation threshold, and to the formation of conductive paths. The statement is based on the comparison dependences of the relative dielectric permittivity (dielectric loss, resp.) and volume resistivity (Figures 4 and 5) on filler content and the mechanism of dielectric loss occurrence [11] related mostly to the nature of dielectric polarization.

4.1.2. Dielectric Loss. The tendencies of the changes in dielectric loss with frequency and filler content (Figure 6) are analogous to those observed in the case of dielectric constant. There also three groups of composites with regard to the filler content. But the dielectric loss value for the composite comprising $30 \mathrm{w} . \%$ of carbon black is not so close to those for the composites comprising 10 and $20 \mathrm{w} . \%$ of carbon black

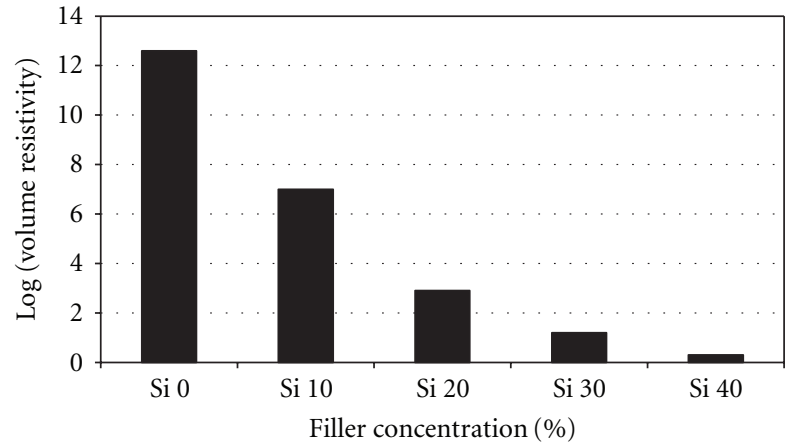

FiguRE 5: Dependence of volume resistivity $(\Omega \cdot \mathrm{m})$ on filler concentration.

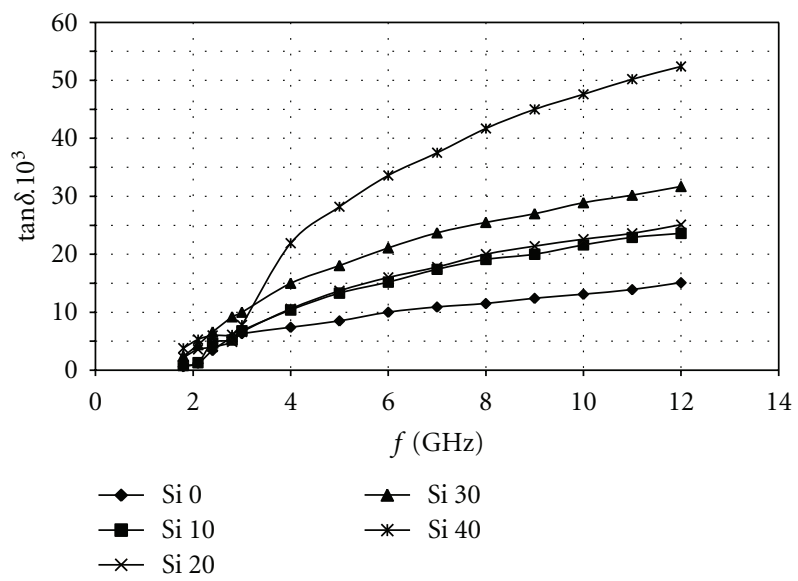

Figure 6: Frequency $(f, \mathrm{GHz})$ dependence of dielectric loss ( $\tan \delta$. $\left.10^{3}\right)$ at various filler content in the composites.

as the dielectric constant values are. That means that the dielectric loss is a parameter more sensitive to the frequency changes and filler content than the dielectric constant. As clarified above the mechanism of occurrence of dielectric losses is related mostly to the nature of dielectric polarization [11]. Since the polarization caused by orientation of the molecule dipoles cannot compensate immediately the fluctuating electrical field strength $E$, a certain relaxation time is necessary. The electrical dipoles cannot compensate for the electrical field by microwave frequencies in the 1$12 \mathrm{GHz}$ range. As a consequence the dielectric permittivity and the dielectric loss increase with increasing frequency. The mobility of the free electrons is the determining parameter for carbon black filled composites. If the mobility of the free electrons along the percolation paths is not high enough to compensate the field (at higher frequencies), the dielectric constant and dielectric loss increase. At higher frequencies the outer field can no longer be compensated because the mobility of the free electrons is not high enough. The consequence is a phase difference resulting in a dielectric loss. The maximum loss occurs if all free electrons are accelerated all the time. This is the case when filler loading reaches and overrides the percolation threshold. It must be emphasized 


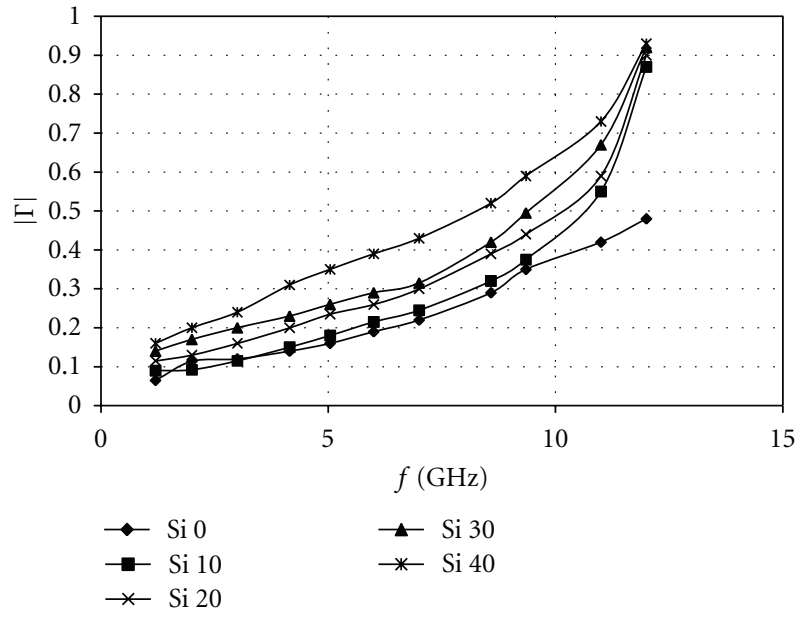

Figure 7: Frequency dependence of the reflection coefficient at various filler content in the composites.

that to reach a very high level of microwave properties, the only way is to override the percolation threshold.

\subsection{Microwave Properties}

4.2.1. Reflection of Electromagnetic Waves. As visible from Figure 7 the reflection of electromagnetic waves from composites surface grows with carbon black content and frequency increase. Following peculiarities are observed in the course of dependences.

(1) In frequency range $1-8 \mathrm{GHz}$, the reflection increasing with frequency increase is almost linear, and the values are very close at $1 \mathrm{GHz}$ then starting to get apart; that is, the impact of filler content becomes more sensitive.

(2) In frequency range $8-12 \mathrm{GHz}$ and especially over $10 \mathrm{GHz}$, reflection increase according to filler content has almost exponential mode (i.e., becomes very drastic) while for nonfilled control sample the dependence is still close to linear.

(3) At frequencies over $11 \mathrm{GHz}$, impact of filler content of filled samples begins again to decrease, and the values of reflection coefficients are getting closer, all of them being within 0.85-0.92 limits.

4.2.2. Attenuation of Electromagnetic Waves. As seen from Figure 8, as whole, attenuation of electromagnetic waves increases also with frequency and carbon black amount increase. The impact of filler content on the attenuation is much stronger in the entire frequency range than the impact on the reflection. Attenuation of sample with $40 \mathrm{w} . \%$ carbon black is the most significant and reaches values over $30 \mathrm{db} / \mathrm{cm}$ at $12 \mathrm{GHz}$, that makes it material applicable for improving electromagnetic compatibility between various units emitting electromagnetic waves. At lower filling rates up to $8 \mathrm{GHz}$, attenuation increases slightly with frequency and follows nearly linear mode while its increase in the range $8-12 \mathrm{GHz}$ is much more speedy. Dependences observed are complying with examined changes of $\varepsilon_{r}$ and $\operatorname{tg} \delta_{\varepsilon}$ and filler content, and can be explained with basic theoretic

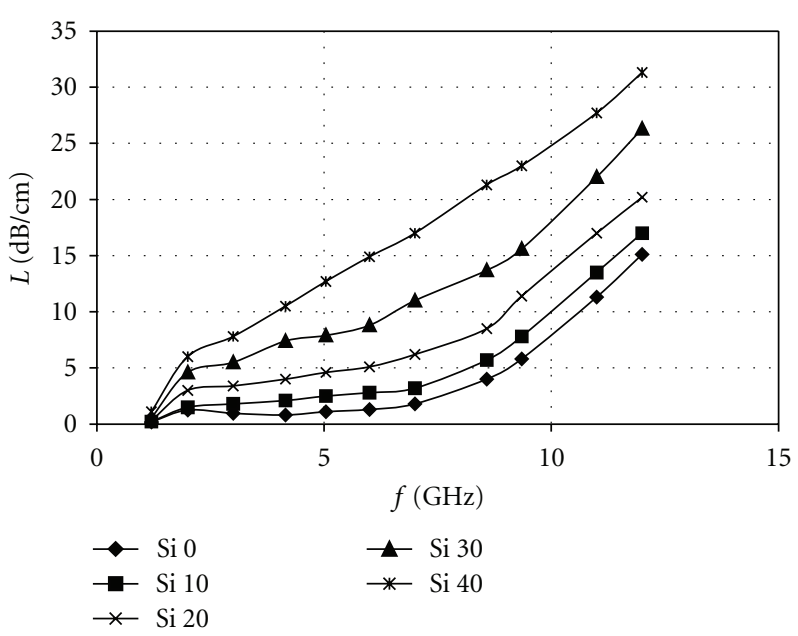

FIGURE 8: Frequency dependence of attenuation of electromagnetic waves at various filler content in the composites.

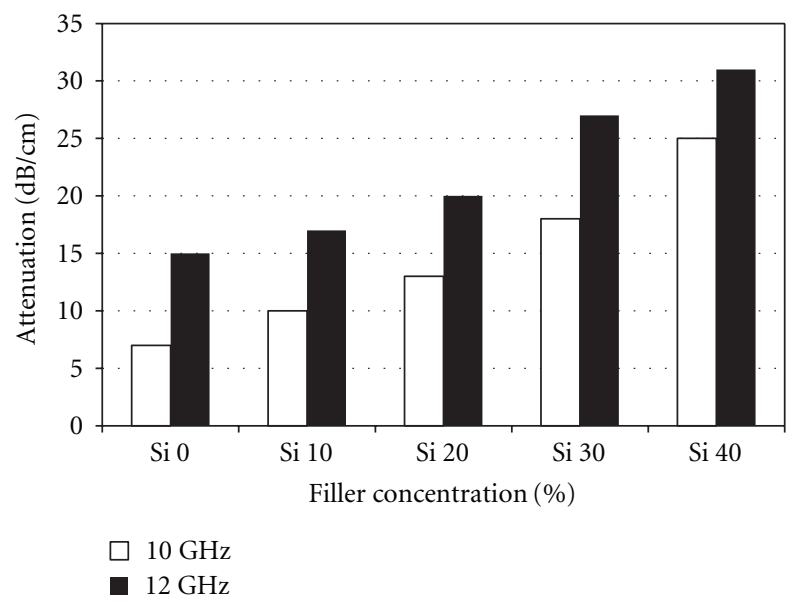

FIGURE 9: Dependence of attenuation of electromagnetic waves on the concentration of the introduced filler.

formulations, describing the interaction of electromagnetic waves with materials having dielectric matrix structure with discretely and uniformly dispersed conductive phase in it [12]. Obviously the increasing of $\operatorname{tg} \delta_{\varepsilon}\left(\operatorname{tg} \delta_{\varepsilon}=\varepsilon^{i i} / \varepsilon^{i}\right)$ with frequency and filling increase is of decisive importance that plays significant role in the improvement of attenuation. At lower frequencies $(1-8 \mathrm{GHz})$ and lower amounts of filler, dielectric parameters of composites, the tangent of dielectric loss particularly, are not high enough to ensure high attenuation of electromagnetic waves (Figure 9).

4.2.3. Shielding Effectiveness. On Figure 10 is shown the changing of shielding effectiveness with frequency and content increase. It is obvious that this changing is of different mode according to filling amount which can be explained by (4) used to calculate shielding effectiveness and described above peculiarities in the changes of reflection and absorption according to frequency and filler content. On that base, quite naturally, shielding effectiveness of composite 


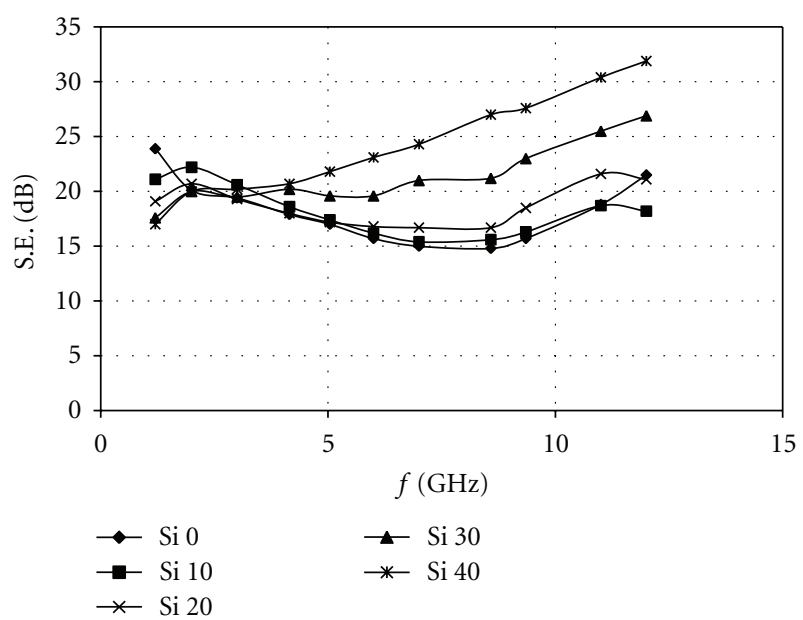

Figure 10: Frequency dependence of shielding effectiveness at various filler content in the composites.

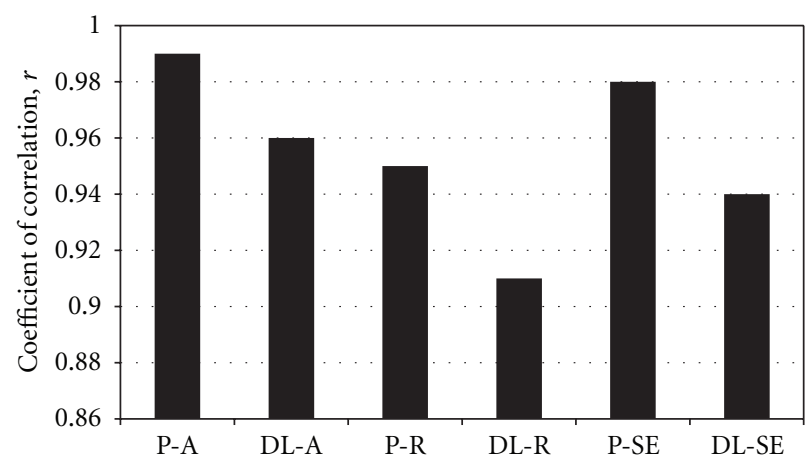

Figure 11: Coefficients of correlation between dielectric and microwave properties: P: dielectric permittivity; A: attenuation; DL: dielectric loss; R: reflection, S.E.: shielding effectiveness.

with 40 w.\% carbon black content increases nearly linear in the whole investigated frequency range after $4 \mathrm{GHz}$. For all other composites, the electromagnetic shielding observed first decreases, followed by a frequency region in which it increases. When increasing filler content, the frequency, at which shielding effectiveness starts to increase, shifts smoothly to lower frequencies that is observed at $9 \mathrm{GHz}$ with nonfilled sample and at $6 \mathrm{GHz}$ with the composite containing $30 \mathrm{w} . \%$. It is obvious that the sample with $40 \mathrm{w} . \%$ carbon black has the highest shielding effectiveness in the frequency range investigated.

\subsection{Correlation between the Dielectric and Microwave Prop-} erties. The correlation between the relative dielectric permittivity and, reflection and attenuation of electromagnetic waves, and shielding effectiveness as well as the correlation between the dielectric loss, and reflection and attenuation electromagnetic waves, and shielding effectiveness has been studied. The following has been established calculating the correlation coefficient (Figure 11).

The calculated values (Figure 11) allow the following conclusions: there is a perfect degree of correlation between the dielectric and microwave properties in all cases investigated. The correlation between the dielectric loss, and reflection and attenuation of electromagnetic waves $(0.91$ and 0.96 , resp.) is a little bit lower than that between the dielectric permittivity and microwave properties (0.95 and 0.99 , resp.). Between the dielectric constant and shielding effectiveness (0.98), and the dielectric loss and shielding effectiveness (0.94) the degree of correlation is also perfect. That reveals that on the basis of dielectric properties data one can predict the microwave properties (values of reflection and attenuation of electromagnetic waves and shielding effectiveness) for the composites studied.

\section{Conclusions}

The dielectric and microwave properties of siloxane rubber composites comprising furnace carbon black filler at various concentrations have been investigated.

(1) It has been established that the increasing frequency and filler content lead to an increase in the relative permittivity and tangent of dielectric loss angle. At higher filler content the effects become more pronounced, especially those upon dielectric loss.

(2) It has been also established that there are two welldistinguished areas in all dependences of microwave properties on frequency and filler content increasing. The first is between 1 and $8 \mathrm{GHz}$ wherein the reflection and attenuation of microwaves do not change considerably with frequency and filler content alternation while shielding effectiveness worsens. The second area is between 8 and $12 \mathrm{GHz}$ wherein the reflection and attenuation of microwaves increase drastically with the increasing frequency and filler content. Shielding effectiveness improves also.

(3) The correlation between the dielectric and microwave characteristics of the composites has been studied. It has been established that in all cases the degree of correlation is perfect. The correlation degree between the dielectric permittivity and the microwave properties is superior to that between the dielectric loss and microwave properties. Between the dielectric constant and shielding effectiveness and the dielectric loss and shielding effectiveness, the degree of correlation is also perfect. That reveals that on the basis of dielectric properties data, one can predict the microwave properties for the composites studied.

\section{Acknowledgments}

The present paper is a result of an international collaboration program between University of Tabuk, Tabuk, Kingdom of Saudi Arabia and the University of Chemical Technology and Metallurgy, Sofia, Bulgaria. The authors gratefully acknowledge the financial support from the University of Tabuk. 


\section{References}

[1] S. K. Kwon, J. M. Ahn, G. H. Kim, C. H. Chun, J. S. Hwang, and J. H. Lee, "Microwave absorbing properties of carbon black/silicone rubber blend," Polymer Engineering and Science, vol. 42, no. 11, pp. 2165-2171, 2002.

[2] S. Thomas and R. Stephen, Eds., Rubber Nanocomposites, Wiley, Singapore, 2010.

[3] J. Schmidt, M. Boehling, U. Burkhardt, and Y. Grin, "Preparation of titanium diboride $\mathrm{TiB}_{2}$ by spark plasma sintering at slow heating rate," Science and Technology of Advanced Materials, vol. 8, no. 5, pp. 376-382, 2007.

[4] B. Basu, G. B. Raju, and A. K. Suri, "Processing and properties of monolithic TiB2 based materials," International Materials Reviews, vol. 51, no. 6, pp. 352-374, 2006.

[5] S. E. Bates, "Synthesis of titanium boride $\left(\mathrm{TiB}_{2}\right)$ nanocrystallites by solution-phase processing," Journal of Materials Research, vol. 10, no. 10, pp. 2599-2612, 1995.

[6] P. Dick, Rubber Technology, Wiley, New York, NY, USA, 2005.

[7] C. R. Paul, Introduction to Electromagnetic Compatibility, John Wiley \& Sons, New York, NY, USA, 1992.

[8] B. Meng, J. Booske, and R. Cooper, "Extended cavity perturbation technique to determine the complex permittivity of dielectric materials," IEEE Transactions on Microwave Theory and Techniques, vol. 43, no. 11, pp. 2633-2636, 1995.

[9] J. Cohen and P. Cohen, Applied Multiple Regression/Correlation Analysis, Lawrence Erlbaum Associates, Hillsdale, NY, USA, 1983.

[10] S. Hamdam, D. Hashim, and M. Yusop, "Dielectric studies of some rubber materials at microwave frequencies," Asean Journal for Science and Technology Development, vol. 21, no. 1, pp. 69-79, 2004.

[11] W. Niedermeier, "Degussa," Technical Reports TR 832.

[12] N. Dishovsky, "Rubber based composites with active behavior to microwaves," Journal of the University of Chemical Technology and Metallurgy, vol. 44, no. 2, pp. 115-122, 2009. 

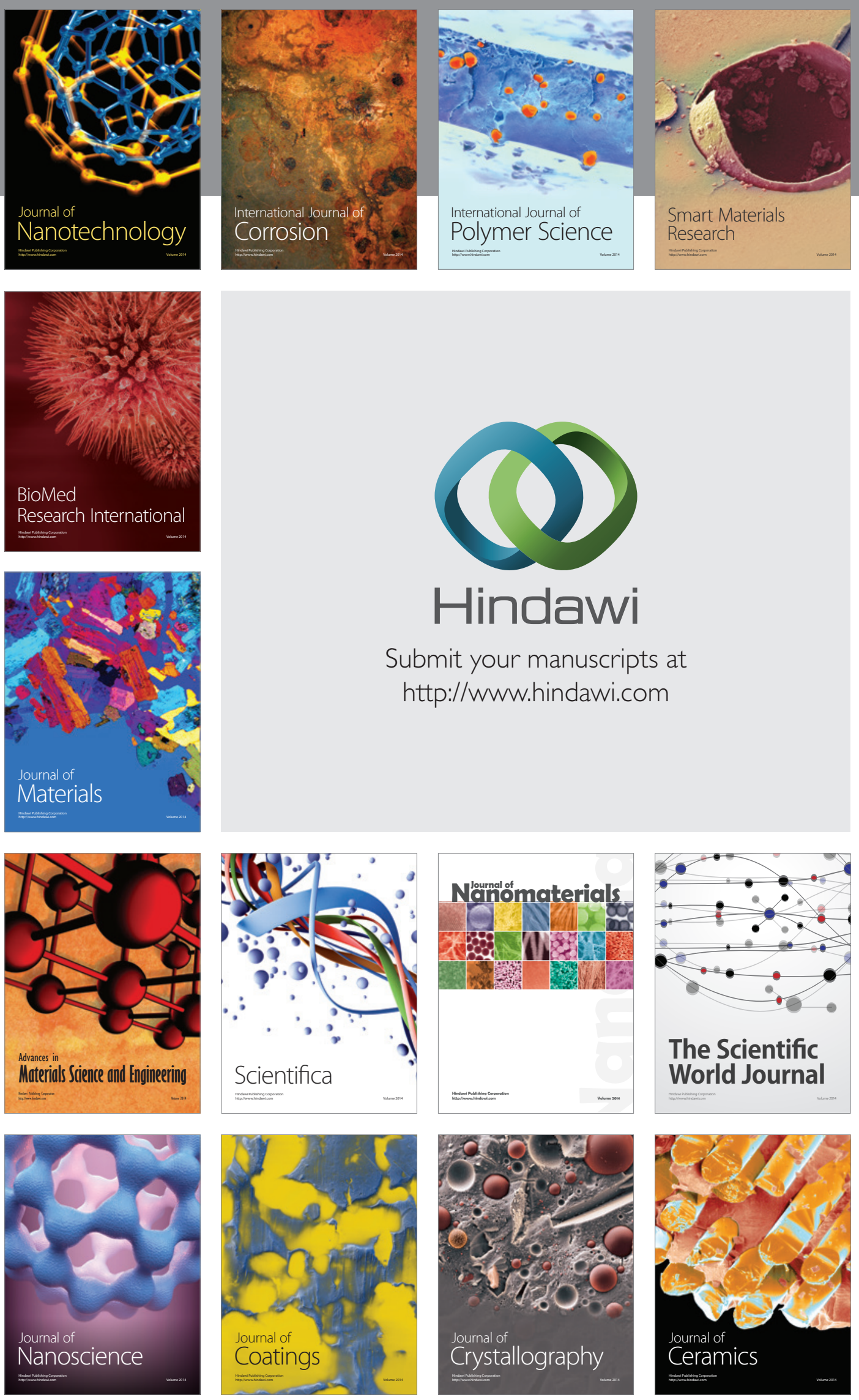

The Scientific World Journal

Submit your manuscripts at

http://www.hindawi.com

\section{World Journal}

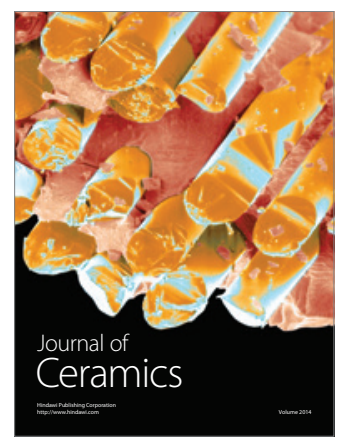

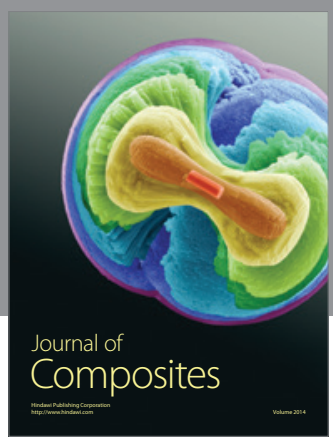
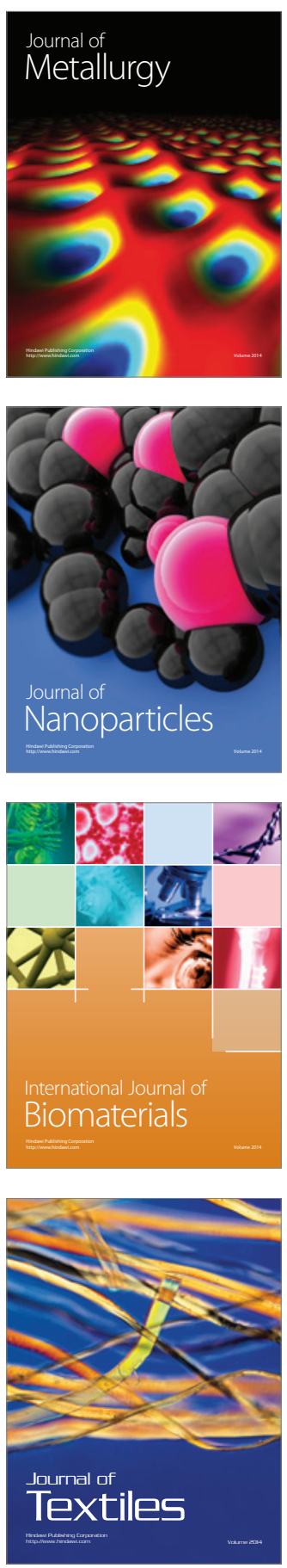